\section{PCR-based assay for mating type and diploidy in Chlamydomonas}

\author{
Ivan Zamora, Jessica L. Feldman, and Wallace F. Marshall \\ University of California, San Francisco, CA, USA
}

BioTechniques 37:534-536(October 2004)
The unicellular green alga Chlamydomonas reinhardtii has become an important genetic model system for studying photosynthesis, cell motility, and organelle biogenesis (1). Chlamydomonas has many of the same advantages as budding yeast for doing genetics, including rapid growth, the ability to maintain both haploids and diploids, tetrad analysis, easy transformation with DNA, and a completely sequenced genome. Because of these advantages, Chlamydomonas is often referred to as "green yeast."

The primary strength of Chlamydomonas is its powerful genetics. During most genetic procedures, it becomes necessary to determine the mating type of a given strain. Chlamydomonas is a heterothallic organism, with two mating types denoted $\mathrm{mt}+$ and $\mathrm{mt}$-. Mating type is determined by two different alleles of a single MT locus, and unlike budding yeast, there are no additional silent copies in the genome. Mating type is normally assessed using functional mating tests with tester strains (2). These functional mating tests often fail because many mutants of interest (e.g., mutants with defective flagellar motility) are very poor maters. Moreover, mating efficiency can be affected by variation in media or precise growth conditions. Thus there is a need for a rapid method to determine mating type that will work even in mutants with low mating efficiency.

A related problem concerns testing for diploidy. Diploid strains are extremely valuable because they allow complementation tests and the determination of whether a given mutation is recessive or dominant. One selects for diploids by mating cells that contain combinations of closely linked auxotrophic markers such that a diploid, but neither haploid parent strain, can grow on minimal media. However, both spontaneous revertants for either marker, or recombination occurring between the linked markers, can result in haploid cells that still grow on minimal media. It is therefore always critical to verify that a putative diploid strain is actually a diploid. Some workers have reported the analysis of diploids through the use of Southern blot analysis, either with probes within the MT locus (3) or with probes outside the MT locus that detect an restriction fragment-length polymorphism (RFLP) within MT that distinguishes the two alleles (4). However, Southern blot analysis is time-consuming and inconvenient for analyzing a large number of strains.

We have developed a PCR-based method for assaying mating type and for distinguishing haploids from dip- loids. This method takes advantage of the fact that the $\mathrm{mt}+$ and $\mathrm{mt}-$ alleles of the MT locus each contain unique sequences not present in the other allele. The use of PCR allows us to avoid Southern blot analysis steps. A PCRbased method for detecting the $\mathrm{mt}+$ and mt- alleles has been reported (5). However, it was reported that this method falsely classified some known diploid cells as haploid (5), calling the reliability of the method into question. This previously described method required two steps, a resin-based genomic DNA purification followed by a PCR step.

We have developed a new one-step PCR-based approach that circumvents these difficulties. Based on the $\mathrm{mt}+$ and mt- specific allele sequences $(6,7)$ we designed four primer pairs specific to each allele (Table 1). PCR was carried out using the conditions listed below, with DNA isolated from wild type mt+ or mt- cells. As illustrated in Figure 1A, all four $\mathrm{mt}+$ specific primer pairs gave products of the expected size when used in reactions with DNA from mt+ cells. In contrast, none of the $\mathrm{mt}+\mathrm{spe}-$ cific primers gave any detectable product with DNA from mt- cells. Likewise, all four mt- specific primer pairs gave products of the expected sizes when used in reactions with DNA from mt-

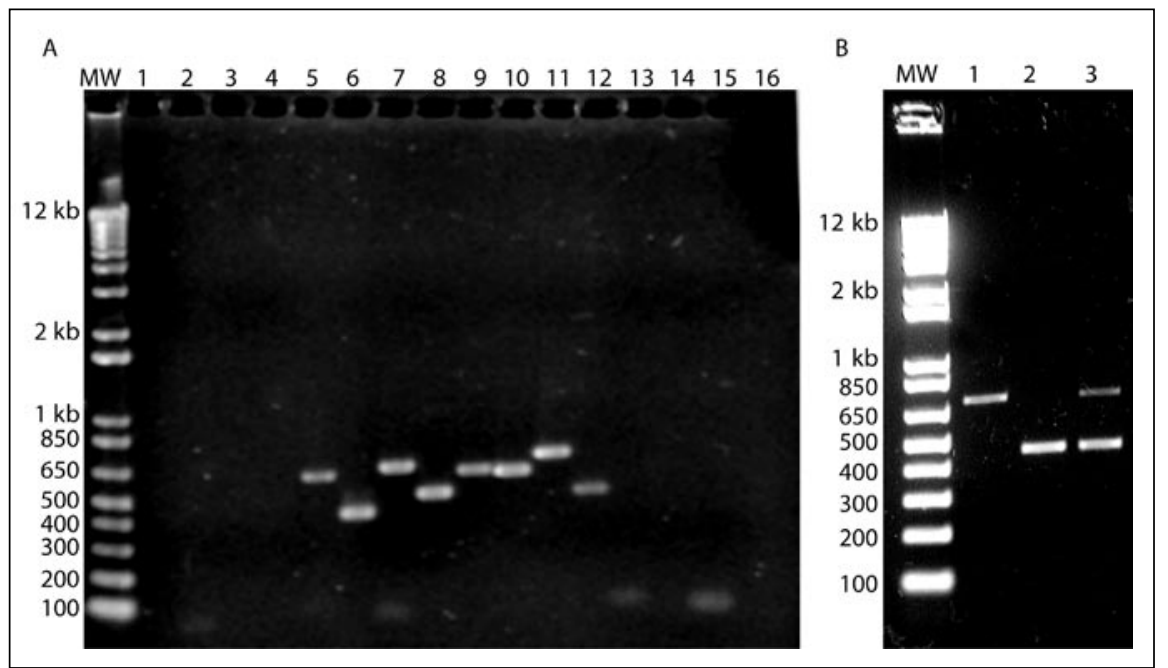

Figure 1. PCR analysis of MT locus. (A) Specificity of primer pairs. PCRs performed using DNA from either $\mathrm{mt}+$ cells (lanes 1-8) or mt- cells (lanes 9-16). Primer pairs used in individual reactions were MTM1 (lanes 1 and 9), MTM2 (lanes 2 and 10), MTM3 (lanes 3 and 11), MTM4 (lanes 4 and 12), MTP1 (lanes 5 and 13), MTP2 (lanes 6 and 14), MTP3 (lanes 7 and 15), and MTP4 (lanes 8 and 16). Primers designed to recognize $\mathrm{mt}+$ locus give no products with DNA from mt- cells, and vice versa. MW represents DNA molecular size ladder (1-kb Plus Ladder; Invitrogen, Carlsbad, CA, USA). (B) Example of single-colony PCR analysis of mating type and diploidy. Lane 1, haploid mt- strain cc-124; lane 2, haploid $\mathrm{mt}+$ strain cc-125; lane 3 , diploid strain containing both $\mathrm{mt}+$ and $\mathrm{mt}-$ alleles. 
Table 1. PCR Primer Pairs for Chlamydomonas Mating Type Analysis

\begin{tabular}{|c|c|c|c|}
\hline Name & Sequence & $\begin{array}{c}\mathbf{T}_{\mathrm{m}} \\
\left({ }^{\circ} \mathbf{C}\right)\end{array}$ & $\begin{array}{l}\text { Size } \\
\text { (bp) }\end{array}$ \\
\hline MTM1F & 5'-CTGCTGGTACAAAGGTGTGGCACG-3' & 70.0 & \multirow[t]{2}{*}{607} \\
\hline MTM1R & 5'-CGGGTGCAGAGTAGCTGTGACCCG-3' & 73.1 & \\
\hline MTM2F & 5'-GCCGCTGCCGCACGTCATCATGTG-3' & 79.4 & \multirow[t]{2}{*}{592} \\
\hline MTM2R & 5'-CATGCAGTCTCTCACCCATTCGGC-3' & 71.3 & \\
\hline MTM3F & 5'-CGACGACTTGGCATCGACAGGTGG-3' & 74.0 & \multirow[t]{2}{*}{689} \\
\hline MTM3R & 5'-CTCGGCCAGAACCTTTCATAGGGTGG-3' & 72.1 & \\
\hline MTM4F & 5'-CCACCCTATGAAAGGTTCTGGCCGAG-3' & 72.1 & \multirow[t]{2}{*}{479} \\
\hline MTM4R & 5'-GCGCCACCAGCCAAGTTATTGACG-3' & 72.4 & \\
\hline MTP1F & 5'-GCCGCAAATCATGTCAATATAGCCTGG-3' & 70.6 & \multirow[t]{2}{*}{611} \\
\hline MTP1R & 5'-CGCACATGAGACGTTACAGCGGTTG-3' & 71.7 & \\
\hline MTP2F & 5'-GCTGGCATTCCTGTATCCTTGACGC-3' & 70.8 & \multirow[t]{2}{*}{423} \\
\hline MTP2R & 5'-GCGGCGTAACATAAAGGAGGGTCG-3' & 70.3 & \\
\hline MTP3F & 5'-GCTGCACAATTCGTCTTGGCTGGTG-3' & 72.8 & \multirow[t]{2}{*}{644} \\
\hline MTP3R & 5'-CTGGCCTCGCCTTCACAAGAGAGC-3' & 71.6 & \\
\hline MTP4F & 5'-GCTTGAACGCTGCAGCTATGACGGC-3' & 73.6 & \multirow[t]{2}{*}{498} \\
\hline MTP4R & 5'-GCGTCACAGCCTCGTAAGCCGTAC-3' & 70.1 & \\
\hline
\end{tabular}

cells and gave no product in reactions with DNA from mt+ cells. This establishes that the primer pairs are specific for the MT region.

We then selected one $\mathrm{mt}+$ specific primer pair and one mt- specific primer pair, which give different size products, allowing both primer pairs to be combined in a single PCR. The mt+ allele was detected using the pair of primers

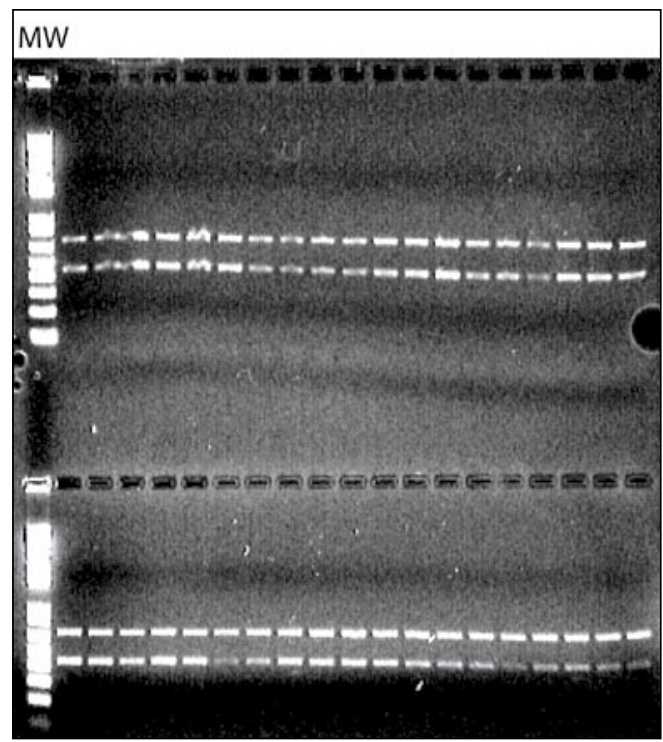

Figure 2. Reliability analysis of diploid detection. Thirty-eight independently generated $\arg 2 /$ arg 7 diploids were subjected to PCR-based $\mathrm{mt}$ analysis. One hundred percent of diploids showed the expected two bands. MW represents DNA molecular size ladder (Invitrogen).
MTP2F and MTP2R (Table 1), which were designed to detect a 423-bp piece of the mt+ specific gene FUS1 (GenBank $^{\circledR}$ accession no. U49864) and which encodes a mating type plus specific glycoprotein (6). The mt- allele was detected using the pair of primers MTM3F and MTM3R (Table 1), which were designed to detect a 689-bp piece of the mt- specific gene MIDI (GenBank accession no. U92071, also known as IMP11) and which entranscription factor involved in sex determination (7). PCRs using MTM3F and MTM3R with DNA isolated from the strain cf181, an mt- strain carrying a deletion of the MID1 gene, gave no detectable product (data not shown), further confirming the specificity of this primer pair.

PCR analysis of the MT locus was performed as follows. Single colonies of cells from a plate were suspended in $5 \mu \mathrm{L}$ of water and boiled for $5 \mathrm{~min}$ in a PCR tube. Into this same tube, the four primers MTP2F, MTP2R, MTM3F, and MTM3R (38 pmol of each) were added along with nucleotides, polymerase mixture, buffer (both from BD Biosciences Clontech, Palo Alto, CA, USA), and water to a final codes a putative leucine-zipper reaction volume of $25 \mu \mathrm{L}$, according to manufacturer's instructions. PCRs were performed using the Advantage ${ }^{\circledR} \mathrm{GC}$ kit (BD Biosciences Clontech). Reactions were run for 30 amplification cycles, each consisting of denaturation at $94^{\circ} \mathrm{C}$ for $30 \mathrm{~s}$, followed by annealing/elongation at $68^{\circ} \mathrm{C}$ for $1.5 \mathrm{~min}$. Figure $1 \mathrm{~B}$ illustrates the application of this assay to wild-type test strains, and confirms that we can distinguish haploid $\mathrm{mt}+$, haploid mt-, and diploid cells. Diploid cells are easily distinguished by the presence of two bands.

In order to check the reliability of diploid detection in our procedure, we isolated 38 independently generated diploid strains (formed by mating arg $2 m t+$ with $\arg 7 m t$ - and selecting for $\arg 2 / \arg 7$ diploids that can grow on media lacking arginine due to intragenic complementation between the arg2 and $\arg 7$ alleles). These diploid lines were subjected to PCR analysis (Figure 2 ), which indicated that, out of 38 diploid lines, all showed the expected pair of products.

In contrast to previously described mating type assays, PCR-based assays are easily applied to large numbers of strains and to strains that mate poorly. The procedure documented here represents a significant advance over that reported by Werner and Mergenhagen (5), in that our method $(i)$ eliminates the need for a separate DNA isolation step and (ii) detects diploids with $100 \%$ reliability.

\section{COMPETING INTERESTS STATEMENT}

The authors declare no conflicts of interest.

\section{REFERENCES}

1.Lefebvre, P.A. and C.D. Silflow. 1999. Chlamydomonas: the cell and its genomes. Genetics 151:9-14.

2.Harris, E.H. 1989. The Chlamydomonas Sourcebook. Academic Press, San Diego, CA

3.Palombella, A.L. and S.K. Dutcher. 1998. Identification of the gene encoding the tryptophan synthase beta-subunit from Chlamydomonas reinhardtii. Plant Physiol. 117:455464.

4.Wilkerson, C.G., S.M. King, A. Koutoulis, G.J. Pazour, and G.B. Witman. 1995. The 
78,000 M(r) intermediate chain of Chlamydomonas outer arm dynein is a WD-repeat protein required for arm assembly. J. Cell Biol. 129:169-178.

5.Werner, R, and D. Mergenhagen. 1998. Mating type determination of Chlamydomonas reinhardtii by PCR. Plant Mol. Biol. Reporter 16:295-299.

6.Ferris, P.J., J.P. Woessner, and U.W. Goodenough. 1996. A sex recognition glycoprotein is encoded by the plus mating-type gene fus 1 of Chlamydomonas reinhardtii. Mol. Biol. Cell 7:1235-1248.

7.Ferris, P.J. and U.W. Goodenough. 1997. Mating type in Chlamydomonas is specified by mid, the minus-dominance gene. Genetics 146:859-869.

Received 26 April 2004; accepted 9 June 2004.

Address correspondence to Wallace F. Marshall, Department of Biochemistry and Biophysics, GH-N372F Genentech Hall, Box 2200, University of California, San Francisco, 600 16th St., San Francisco, CA 94143, USA. e-mail: wmarshall@biochem. ucsf.edu

\title{
Microarray approach for cloned library quality assessment and the comprehensive identification of positives from library screens
}

\author{
Ronald K. Blackman and Benjamin S. Amidon \\ Millennium Pharmaceuticals, Cambridge, MA, USA \\ BioTechniques 37:536-540 (October 2004)
}

Microarrays that assay RNA expression levels provide a rapid and comprehensive survey of the cellular transcriptome, and their use for expression profiling has become routine for many laboratories $(1,2)$. Conveniently, these same arrays, which frequently assess most or all genes of the genome, can also be used for additional molecular biology applications that require the monitoring of the genes represented in a sample. Cloned DNA libraries are used in many experimental approaches, including yeast two-hybrid experiments, overexpression resistance/suppression studies, and cDNA library screens. Two common concerns for experiments with such libraries are $(i)$ the quality and complexity of the starting library and (ii) whether all possible positives from the screen have been identified. Herein, we show that microarrays designed for RNA expression analysis provide a quick and efficient means to address these issues.

For any set of experiments employing a cloned DNA library, it is critical to know, prior to the actual screen, that the chosen library contains a complete and true representation of the sequences desired for the assay. For most approaches, knowing which genes are present in the library is sufficient for this quality control step, and microarrays can quickly produce such information.

As an example, we desired to know the coverage (complexity) of a commercial human spleen cDNA library (BD Biosciences Clontech, Palo Alto, CA, USA) before we began experiments using it as the "prey" constructs in multiple yeast two-hybrid screens. Our approach was to biotin-label the library DNA in vitro and hybridize it to a GeneChip ${ }^{\circledR}$ (Affymetrix, Santa Clara, CA, USA) that is normally used to expression profile human RNAs. In a best case scenario, we would have compared the representation of the cloned cDNA to the signals produced by the original RNA analyzed in parallel. With this commercial library, the original RNA sample was unavailable, but we could still evaluate the minimum number of coding regions represented in the library. We chose to label the library DNA using random-primed synthesis that incorporated biotinylated nucleotides directly. Alternatively, we could have chosen PCR using biotinylated primers or nucleotides, but this approach might have selected against some classes of inserts (e.g., long inserts) and has other complications (see below).

The library DNA (400 ng) was labeled in a $100-\mu \mathrm{L}$ reaction using the BioPrime ${ }^{\circledR}$ DNA Labeling System (Invitrogen, Carlsbad, CA, USA) following the manufacturer's protocol, except that we labeled for $2 \mathrm{~h}$ and used only $4 \mu \mathrm{L}$ of stop buffer at the end of the reaction. We ran $5 \mu \mathrm{L}$ of the material on a $4 \%$ polyacrylamide gel to monitor the DNA labeling and confirm the manufacturer's estimate of 2-5 $\mu \mathrm{g}$ of synthesized product. After ethidium bromide staining, the newly synthesized DNA appeared as a strongly stained smear between 40 and $140 \mathrm{bp}$. An added benefit of using the random-primed labeling protocol is that these fragments are appropriately sized to provide a good population of targets for the microarray hybridization (3). This could be a significant issue if intact (i.e., much larger) labeled PCR products are used directly.

For the chip hybridization, $80 \mu \mathrm{L}$ of the labeling reaction were brought to a total volume of $305 \mu \mathrm{L}$ in the standard hybridization solution recommended by Affymetrix. (In the preparation of the hybridization cocktail, we considered the $80 \mu \mathrm{L}$ BioPrime reaction as if it were contributing only water to the mixture). The U133A GeneChip was pretreated, hybridized $\left(45^{\circ} \mathrm{C}\right.$ for $\left.16 \mathrm{~h}\right)$, and washed using the manufacturer's procedure typically used for RNA profiling analysis (3). The chip was scanned, and the data was processed by the MAS 5 software (Affymetrix) using the default settings for the analysis. For the spleen library hybridization shown in Figure 1, the software called one-third $(33.7 \%)$ of the genes represented on the chip as "P" (present). While this is a respectable representation, it is somewhat lower than the percentages obtained from typical tissue-specific RNA profiling experiments (4). However, the overall signals we obtained in our hybridizations are also somewhat lower, indicating that our protocol using a few micrograms of double-stranded DNA for 